(*) Tortula laevipila (Brid.) Schwaegr. var. meridionalis (Schimp.) Wijk.

Santiago de Compostela 29TNJ3847. Epífita de Salix sp. (SANT-bryo. 2412).

Tortula papillosa Wills.

Santiago de Compostela 29TNJ3847. Epífita de Robinia Pseudoacacia. (SANT-bryo. 2426). Se confirma la presencia de esta especie en Galicia de la que se conocía una única cita debida a Casares (1915). Novedad para la provincia de Lugo.

\title{
BIBLIOGRAFÍA
}

CASARES-GIL, A. -1915- Enumeración y distribución de las Muscíneas de la Península Ibérica. Trab. Mus. Nac. Cien. Nat. Ser. Bot., 8:1-179. Madrid.

COLMEIRO, M. -1867-Enumeración de las Criptógamas de España y Portugal. Rev. Progresos Cienc., 16-17:54-519. Madrid.

LUSIER, A. -1918- Fragments de Bryologie Ibérique. 14. Mousses de Galice. Broteria Ser. Bot, 16:123-142. Braga.

MERINO, P. -1916- Contribución a la Muscología de la Península Ibérica. Bol. R. Soc. Esp. Hist. Nat., 16:270-276. Madrid.

POTIER DE LA VARDE, R. -1945- Liste des espèces du Genre Fissidens récoltées dans la Péninsule Ibérique par M. et Mme. Allorge. Rev. Bryol. Lichénol, 15:30-39. Paris.

REINOSO, J. -1984- Contribución al conocimiento de la flora briológica de Galicia. Briófitos de la fraga de Caaveiro (La Coruña). I. Musgos. Lazaroa, 6:237-247. Madrid.

REINOSO, J. y J. ÁLVAREZ - 1984- Estudio briológico de la cuenca del río de Rojos (Santiago). Acta Científica Compostelana, 21:273-284. Santiago de Compostela.

(Aceptado para su publicación en enero de 1991)

Dirección de los autores: Departamento de Biología Vegetal (Botánica). Facultad de Biología. Universidad de Santiago. 15706. Santiago de Compostela.

\section{NUEVA LOCALIDAD DE ASTRAGALUS NITIDIFLORUS JIMÉNEZ Y PAU EN ESPAÑA}

Palabras clave: Corología, Astragalus, Fabaceae.

Francisco M. VÁZQUEZ, Eugenio DOMÍNGUEZ y Juan Antonio DEVESA

Al estudiar el género Astragalus en la región extremeña se han detectado unas poblaciones en el $\mathrm{S}$ de la provincia de Badajoz cuya identificación no resulta fácil mediante las floras al uso.

Siguiendo la monografía de Bunge (1868 \& 1869) sobre Astragalus, y considerando el carácter anual de nuestras plantas, los individuos pacenses pertenecen 
sin duda al subgénero Trimeniaeus, y de acuerdo con las características florales y de los tricomas a la sección Platyglottis. Dentro de ella nuestras plantas se ajustan a $A$. verrucosus Moris, pues el carácter connado de las estípulas constituye un carácter muy bueno que no ofrece lugar a dudas. De él indica Bunge (1869) que sólo es conocido de Cerdeña, donde fue descrito por Moris en 1827, así como su estrecho parecido con A. tuberculosus DC.

En el tratamiento dado al género por Chater (1968) A. tuberculosus es incluido como un sinónimo taxonómico de $A$. suberosus Banks \& Solander, mientras que $A$. verrucosus es asociado a éste como taxon afín cuyo status requiere confirmación, aunque expresamente señala como diferencias entrambos la legumbre más ancha y abruptamente picuda de este último, así como el carácter marcadamente connado de sus estípulas (en más de su mitad), que ya señaló Bunge (1868 \& 1869) para la segregación de $A$. verrucosus de $A$. tuberculosus (= A. suberosus). No obstante, al indicar la distribución aporta cierta confusión, pues para A. tuberculosus señala Cerdeña y el SW de Asia, mientras que para el segundo sólo la localidad clásica indicada por Moris (Cerdeña; 1827).

En la revisión de Chater (1968) no se menciona Astragalus nitidiflorus, taxon descrito por Jiménez y Pau (in Pau, 1910) para Cartagena (Murcia, España). Entre los mejores caracteres diferenciales que citan sus descriptores (Pau, 1.c.) destacan el carácter anual de estas plantas, su inflorescencias laxas (floribus spicatibus laxiusculis), la corola amarilla (... petalis luteis, nitidis, vexillo elongato lineari bilobo ...) y la morfología de la legumbre, navicular, con un surco profundo y dorso verrucoso (... leguminibus navicularibus, villosulis, late sulcatis, dorso lacunoso-verrucosis ...)

Mientras que todos los autores recientes parecen estar de acuerdo en que $A$. suberosus y A. tuberculosus son dos nombres para un mismo taxon (Chater, 1968; Chamberlain \& Matthews, 1969; Greuter et al., 1989; Podlech, 1990), no ocurre lo mismo con la posición de A. nitidiflorus y A. verrucosus, considerados por algunos autores como sinónimos (Fernández Casas, 1974; Smythies, 1984), y por Greuter et al. (1.c.) como táxones diferentes: el primero endémico de España y el segundo de Cerdeña.

En favor de este último criterio y a pesar del estrecho paralelismo morfológico en lo concerniente a las estípulas, debe aducirse el color de las flores en A. verrucosus, violetas o purpúreas, tal y como indicó Moris en su descripción original y recalcó Eig (1955), un carácter que no cumplen las plantas recolectadas por nosotros.

Al estudiar el tipo de A. nitidiflorus Jiménez y Pau que se conserva en el herbario del Real Jardín Botánico de Madrid (MA 66838), se ha podido comprobar que las plantas pacenses no difieren sensiblemente del tipo, si bien el carácter emarginado del estandarte que indica Pau (1910) no ha podido confirmarse en el material que se conserva (en fructificación), un carácter que no cumplen las plantas extremeñas, cuyas flores poseen estandarte agudo y entero. No obstante, pensamos que las poblaciones del S de Badajoz constituyen nuevas localidades a considerar de A. nitidiflorus, cuya área de distribución en nuestra Península se restringe, según los conocimientos actuales, a poblaciones murcianas, abulenses y pacenses. 


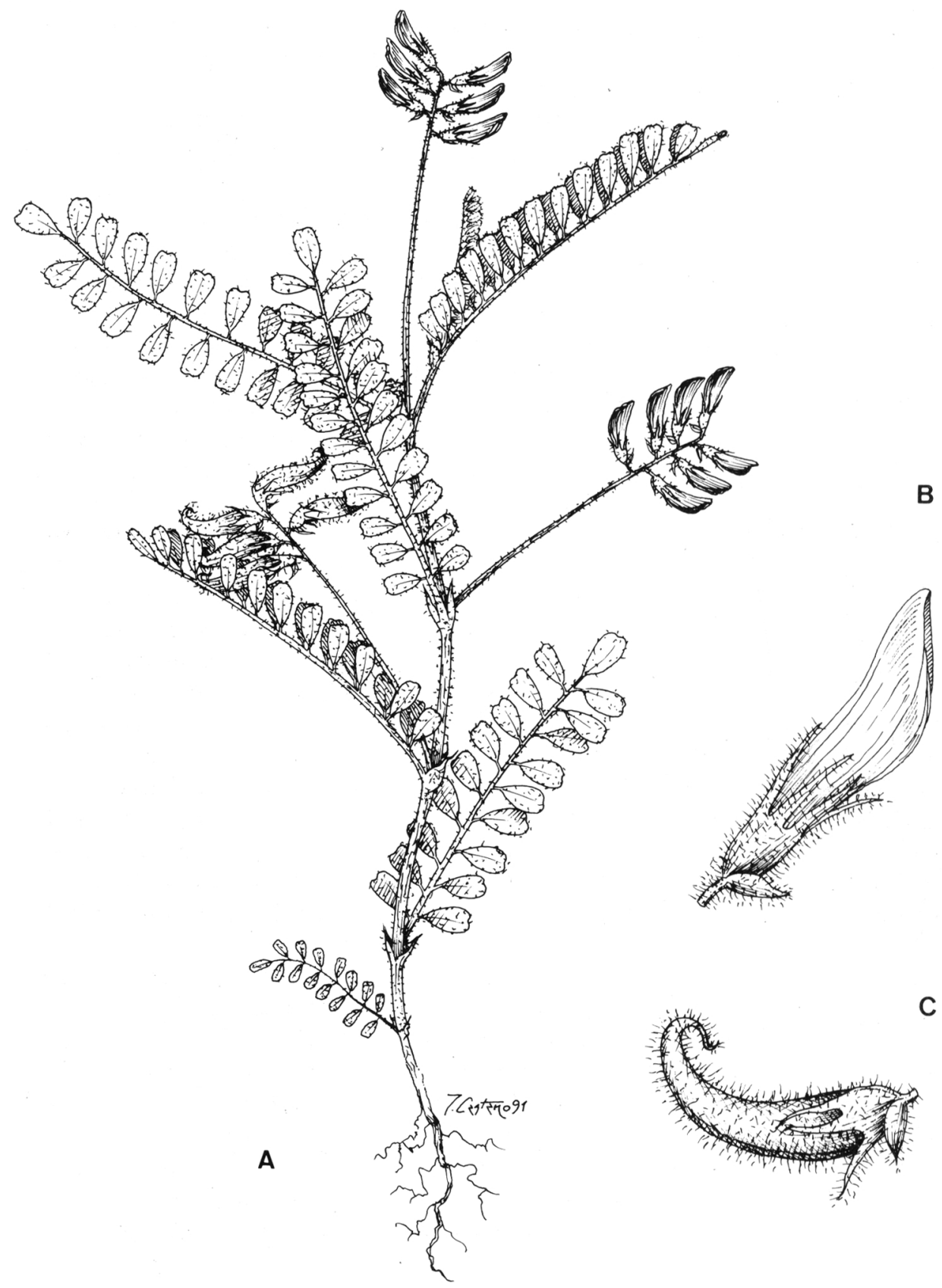

Figura 1. A, aspecto general de Astragalus nitidiflorus Jiménez y Pau. B, detalle flor. C, detalle legumbre. 
Astragalus nitidiflorus Jiménez y Pau, Bol. Soc. Aragon. Ci. Nat., 9:130 (1910) (Fig. 1)

A. verrucosus auct. hisp. non Moris, Stirp. Sard. Elench. 1:12 (1827).

Anual (bienal), herbácea, con tallos decumbentes de $15-45 \mathrm{~cm}$, vilosos, con pelos basifijos blancos salvo en las proximidades de los dos nudos, donde son negros. Hojas de (3,5-)6-9(-11) cm, imparipinnadas, generalmente con 9-15 pares de folíolos marcadamente vilosos en el envés y glabros o con pelos dispersos en el haz; estípulas de (3-)5-11 (-15) mm, anchamente connadas, vilosas por el envés y glabras en el haz. Inflorescencias con 3-7 flores, racemosas, sobre pedúnculos de (3-) 5-8 (-11) cm, vilosos. Cáliz de 8-12 mm, viloso, con pelos basifijos negros; dientes de (3-) 4-6 (-7) mm, subiguales. Corola de (12-) 15-19 (-22) mm, glabra, amarillenta o de color crema; estandarte con ápice agudo, con alguna mácula purpúrea poco conspicua. Legumbre de (15-) 17-22 (-25) mm, arqueada y con pico curvado, verrucosa y vilosa, recorrida por un surco medio que se hace más profundo a medida que madura. Florece de Abril a Mayo.

Ind. loc.: «Cartagena (Franc. de P. Jiménez)».

Lectótipo: Caroli Pau Herbarium Hispanicum / «Astragalus nitidiflorus Jimenez y Pau» (manuscr. Pau) / in Soc. arg. de Cien. Naturales (manuscr. Pau / Legi ... (impr.) / MA 66838, ejemplar izquierdo)

Numero cromosómico.- 2 n=16 (Fig. 2). No conocemos ningún recuento anterior para el taxon.

Ecología y distribución: En tomillares de suelos básicos. Endémico de Avila, $\mathrm{S}$ de Badajoz y E de Murcia.

Material estudiado.- AVILA. Carretera de Avila a Piedrahita, Valle de Anblés, 23.VI.1974, G. López y E. Valdés Bermejo (sec. Podlech, 1990). BADAJOZ. La Parra, ladera Sur de la Sierra de La Parra, 29SQD15, 23-IV-1991, 650 msm, F.M. Vázquez (UNEX 11856). MURCIA. Cartagena, F.P. Jiménez (MA 66838).

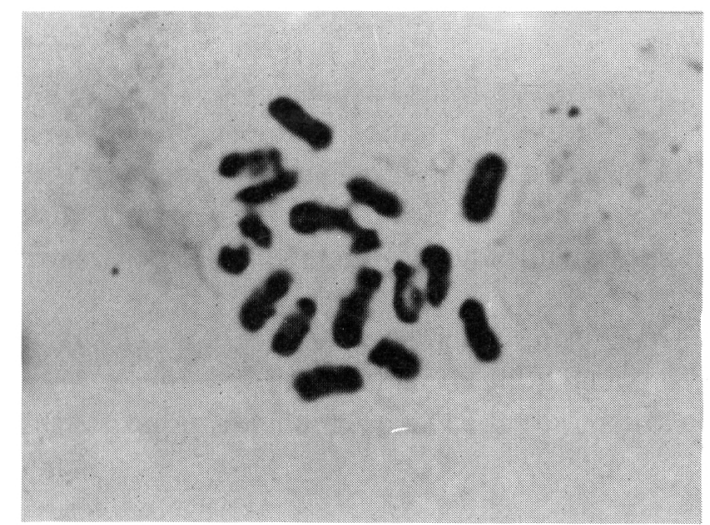

Figura 2.- Metafase somática de Astragalus nitidiflorus Jiménez y Pau (2n=16). 


\section{BIBLIOGRAFÍA}

BUNGE, A. -1868- Generis Astragali Species gerontogeae. Pars prior. Claves diagnosticae. Mem. Acad. Imp. Sci. St.-Pétersb., sér. 7, 11(16):1-140.

BUNGE, A. -1969-Generis Astragali Species gerontogeae. Pars altera, specierum enumeratio. Mem. Acad. Imp. Sci. St.-Pétersb., sér. 7, 15(1):1-254.

CHAMBERLAIN, D.F. \& V.A. MATTHEWS -1969- Astragalus. In: P.H. Davis (ed.) Flora of Turkey, 3:49-254. Edinburgh.

CHATER, A.O. -1968- Astragalus. In: T.G. Tutin et al. (eds.) Flora Europaea, 3:108-124. Cambridge.

FERNANDEZ CASAS, J. -1974- De flora hispanica. Candollea, 29:327-335.

EIG, A. -1955- Systematic studies on Astragali of the Near East. Jerusalem.

GREUTER, W., H.M. BURDET \& G. LONG -1989- Med-Cheklist, 4. Dicotyledones (Lauraceae-Rhamnaceae). Genève.

MORIS, G.G. -1827-Stirpium sardoarum Elenchus, 1. Carali.

MORIS, G.G. -1837/1859- Flora Sardoa, 3. vol. Taurini.

PAU, C. -1910- Astragalus nitidiflorus Jiménez et Pau n.sp. Bol.Soc. Aragon. Ci. Nat., 9:130131.

PODLECH, D. -1990- Revision von Astragalus L. sect. Platyglottis Bunge (Leguminosae). Mitt. Bot. Staatssamm. München, 29:541-572.

SMYTHIES, B.E. -1984- Flora of Spain and the Balearic Islands. Cheklist of vascular plants. Englera, 3(2):213-486.

(Aceptado para su publicación en junio de 1991)

Dirección de los autores: F.M. Vázquez y J.A. Devesa: Departamento de Biología y Producción de los Vegetales. Unidad de Botánica. Facultad de Ciencias. Universidad de Extremadura. Avenida de Elvas s/n, 06071-Badajoz. E. Domínguez: Departamento de Biología Vegetal y Ecología. Avenida de San Alberto Magno s/n, 14004-Córdoba. 


\section{FRAGMENTA PALYNOLOGICA BAETICA}

\section{ESTUDIOS PALINOLÓGICOS EN LA SUBFAMILIA ASTEROIDEAE (COMPOSITAE) EN EL SURESTE DE LA PENÍNSULA IBÉRICA}

Palabras clave. Palinología, Asteroideae, Compositae, España.

Gabriel BLANCA, María JacobaSALINAS, ConsueloDÍAZ DE LA GUARDIA y AnaTeresa ROMERO GARCÍA

El gran número de endemismos de la familia Compositae ha permitido la elaboración de una Tesis de Licenciatura de la que se presentan en este trabajo los resultados obtenidos en la subfamilia Asteroideae, concretamente los que conciernen a las tribus Anthemideae y Senecioneae.

En relación con la tribu Anthemideae, Pardo (1985) estudió la morfología polínica de 8 táxones del género Leucanthemopsis (Giroux) Heywood utilizando microscopía óptica y electrónica de barrido, entre ellos L. pectinata (L.) G. López y Ch. E. Jarvis (sub L. radicans (Cav.) Heywood). Tormo \& Ubera (1987) realizaron un estudio global de la tribu en la Península Ibérica utilizando, además, microscopía electrónica de transmisión; en lo que respecta a las especies ibéricas hay que mencionar también los trabajos de Díez (1987) y Benedí (1988) sobre el género Anthemis y Vallés et al. (1988) sobre el género Artemisia; ninguno de estos trabajos tratan alguna de las especies endémicas del sureste peninsular.

Respecto a la tribu Senecioneae, Tormo et al. (1985) y Díez (1987) estudiaron 7 y 11 especies, respectivamente, del género Senecio, y Blanca et al. (1988), 3 especies del sureste peninsular, S. elodes Boiss. in DC., S. nevadensis Boiss. \& Reuter y $S$. quinqueradiatus Boiss. in DC., todos ellos utilizando microscopía óptica y electrónica de barrido.

Para la observación de los granos de polen en el microscopio óptico, se ha empleado el método acetolítico de Erdtman (1960) ligeramente modificado por Hideux (1972). Se realizaron 30 medidas para cada parámetro, obteniendo la media y la desviación típica.

Este trabajo forma parte de los estudios palinológicos sobre especies endémicas del sureste peninsular o cuya distribución ibérica se restringe a dicha área (Proyecto CAICYT PB850388). 